\title{
Influencia del Compromiso Organizacional sobre el Aprendizaje Organizacional de los docentes de la Universidad Pedagógica Nacional "Francisco Morazán"
}

\author{
Elizabeth Santos Flores \\ Dirección de Postgrado \\ Universidad Pedagógica Nacional "Francisco Morazán" \\ esantosf@upnfm.edu.hn \\ Dani Oved Ochoa Cervantez \\ Ciencias Administrativas, UNICAH \\ daniovedochoacervantez@yahoo.com
}

\section{RESUMEN}

Este documento muestra los resultados de la investigación realizada en la Universidad Pedagógica Nacional "Francisco Morazán" para determinar la influencia del Compromiso Organizacional sobre el Aprendizaje Organizacional. La muestra fue de 118 docentes de la sede de Tegucigalpa. Para medir las variables se utilizaron el cuestionario diseñado por Watkins y Marsick y la escala propuesta por Allen y Meyer. La confiabilidad del instrumento se determinó con el coeficiente Alfa de Cronbach, obteniendo 0.912 para la variable Aprendizaje Organizacional y 0.832 para Compromiso Organizacional. Se estableció el coeficiente de determinación de Pearson para medir el efecto de la variable independiente sobre la dependiente, encontrando: $\mathrm{r}=0.718 \mathrm{y}^{2}$ de 0.516 a un valor $p=000<\alpha$ : 0.05 . Los hallazgos sugieren que los docentes comprometidos con la institución contribuyen con los procesos de transformación de la misma y les conduce a aprender y mejorar su capacidad para hacer cosas nuevas.

\section{PALABRAS CLAVE}

Compromiso Organizacional, Aprendizaje Organizacional, Educación Superior, Universidad Pedagógica Nacional "Francisco Morazán"

\begin{abstract}
This document shows the results of the research conducted at the National Pedagogical University "Francisco Morazán" to determine the influence of Organizational Commitment on Organizational Learning. The sample consisted of 118 teachers from the Tegucigalpa campus. To measure variables, the questionnaire designed by Watkins and Marsick and the scale proposed by Allen and Meyer were used. The reliability of the instrument was determined by Cronbach's Alpha coefficient, obtaining 0.912 for the Organizational Learning variable, and 0.832 for Organizational Commitment one.
\end{abstract}

Recibido 1 de septiembre de 2016 /Aceptado 10 de noviembre de 2016 
The Pearson coefficient of determination was established to measure the effect of the independent variable on the dependent one, finding $r=0.718$ and $R^{2}$ of 0.516 to $p=000<\alpha: 0.05$ values. The findings suggest that teachers committed to the institution contribute to the transformation processes and guide themselves to learn and improve their ability to do new things.

\section{KEYWORDS}

Organizational Commitment, Organizational Learning, Higher Education, National Pedagogical University "Francisco Morazán. 


\section{INTRODUCCIÓN}

En la actualidad las instituciones de educación superior se enfrentan a ciertos desafíos que las obligan a crear estrategias necesarias para lograr que los docentes se comprometan y se integren con los objetivos y proyectos de la institución.

Para Meyer y Allen (1987) el compromiso organizacional consiste en la "Orientación afectiva hacia la organización, el reconocimiento de los costos asociados al dejar de laborar en la organización y la obligación moral de permanecer en ella" (p. 11). Durante los últimos años, investigadores como Joo y Park (2010) y Atak (2011) han dedicado sus trabajos a la comprensión de la naturaleza, antecedentes y consecuencias del compromiso organizacional.

Se ha identificado que altos niveles de compromiso en los colaboradores son importantes porque conducen a resultados favorables para la organización (Kamis y Noermijati, 2013). Asimismo, se ha identificado que el compromiso de los colaboradores afecta positivamente otras áreas de la organización, tales como la capacidad que tiene para "aprender continuamente y de transformarse, y la capacidad de organización para hacer algo nuevo todo el tiempo" (Watkins y Marsick, 1993).

Se sugiere que en la medida que los colaboradores muestran altos niveles de compromiso se incrementa la capacidad de la institución para lograr esa capacidad de aprendizaje. No obstante, existen en mayor número las investigaciones sobre el compromiso en el sector comercial, muy poca investigación se ha llevado a cabo en entornos educativos del nivel superior.

La investigación en este contexto cobra relevancia siendo que los maestros que no están comprometidos con su lugar de trabajo podrían realizar un menor esfuerzo en el aula en comparación con los profesores con altos niveles de compromiso, esto afectaría adversamente el aprendizaje de los estudiantes.

El examinar y confirmar la relación entre el compromiso organizacional y aprendizaje organizacional proporcionará apoyo a los directivos para tener una mejor comprensión del comportamiento y las actitudes de los docentes; además, los resultados del estudio proporcionarán las bases para mejorar el compromiso, la creatividad, la innovación y el rendimiento general de la institución.

Finalmente, la identificación de factores que ayudan a fomentar el compromiso y el aprendizaje ayudará a generar políticas que permitan a la UPNFM atraer y retener al personal más calificado. 


\section{DISCUSIÓN TEÓRICA}

\section{Compromiso organizacional}

Aun no existe consenso sobre la definición y la forma en que se mide el compromiso organizacional (Rashid, Sambasivan y Johari, 2003), no obstante, la revisión de la literatura permite identificar una serie de conceptos utilizados para definirlo, la Tabla 1 recoge algunos de ellos.

Tabla 1 Conceptos de compromiso organizacional

\begin{tabular}{cl}
\hline Autor & \multicolumn{1}{c}{ Concepto } \\
\hline $\begin{array}{c}\text { Mowday, Steers y Porter } \\
(1979, \text { p. 226) }\end{array}$ & $\begin{array}{l}\text { "Fuerte creencia y aceptación de los objetivos y valores de la organización, } \\
\text { voluntad de ejercer un considerable esfuerzo en la organización y un fuerte deseo } \\
\text { de permanecer en ella" }\end{array}$ \\
Newstrom y Davies, (2002,p. & $\begin{array}{l}\text { "Grado en que un colaborador se identifica con la organización y desea seguir } \\
\text { participando activamente en ella" } \\
211)\end{array}$ \\
"Actitudes laborales de los colaboradores hacia la organizaciones en donde no sólo \\
Greenberg y Baron, 2003, p. \\
160) \\
$\begin{array}{l}\text { Grado en que un colaborador se identifica con su organización, sino también si el } \\
\text { colaborador está o no dispuesto a salir de su organización" } \\
\text { "Orientación afectiva hacia la organización, el reconocimiento de los costos } \\
\text { asociados al dejar de laborar en la organización y la obligación moral de } \\
\text { permanecer en ella" }\end{array}$
\end{tabular}

Fuente: Elaboración propia

Entre los autores más reconocidos que abordan el compromiso de los colaboradores hacia la organización se encuentran Meyer y Allen (1984), para ellos, el compromiso organizacional se divide en tres tipos, descritos a continuación:

\section{a) Compromiso afectivo}

Se refiere al deseo de un colaborador en continuar trabajando para una organización debido al apego emocional y la participación e identificación que éste mantiene con los objetivos y valores de la organización (Allen y Meyer, 1990; Lee, Allen, Meyer y Rhee, 2001; Meyer, Allen, y Gellatly, 1990).

Así, el apego del colaborador genera el deseo de seguir trabajando para una organización, genera un alineamiento del colaborador con las metas y los valores, implica que si un colaborador tiene un fuerte compromiso afectivo va a permanecer en la organización porque él así lo desea. (Meyer y Allen, 1993; Meyer, Allen y Smith, 1993).

\section{b) Compromiso de continuidad}

Se basa en los costos que están asociados con el abandono de una organización específica: entre menor es el número de alternativas de trabajo para el colaborador, más fuerte es su compromiso de continuidad a su actual organización, es decir, hay un costo grande al dejar la organización. Si un colaborador exhibe un fuerte compromiso de continuidad se va a quedar con su organización porque siente que dejarla tendría un 
costo demasiado elevado (Meyer, Allen, y Gellatly, 1990; Meyer y Allen, 1993; Meyer y Parfyonova, 2010).

\section{c) Compromiso normativo}

Se refiere a la obligación percibida de los colaboradores a permanecer con su organización, aquí los valores sociales pueden ejercer presiones sobre el colaborador, por lo tanto, se quedará con una organización porque sienten que debería ser congruente con sus valores adquiridos (Meyer y Allen, 1993). Los colaboradores que tienen un alto grado de compromiso normativo no se atreven a decepcionar a sus empleadores y se preocupan por lo que sus compañeros pensarían de ellos si dejan la organización.

\section{Aprendizaje organizacional}

Existe una discusión sobre quien fue el primer autor en hablar del término aprendizaje organizacional. Fernández de Morgado (2008) considera que fueron March y Simon en 1958 quienes acuñaron el término. En las últimas décadas son varios los autores que han definido el aprendizaje organizacional, tal como se muestra en la Tabla 2.

Tabla 2 Conceptos de Aprendizaje Organizacional

\begin{tabular}{c|c}
\hline Autor & \multicolumn{1}{c}{ Concepto } \\
\hline $\begin{array}{c}\text { (Watkins y Marsick, 1993, p. } \\
\text { 152). }\end{array}$ & $\begin{array}{l}\text { Una organización de aprendizaje "es aquella que aprende continuamente y se } \\
\text { transforma, se refiere a los cambios de la capacidad de organización para hacer algo } \\
\text { nuevo todo el tiempo" } \\
\text { "Proceso dinámico de creación, adquisición, e integración del conocimiento dirigido } \\
\text { al desarrollo de los recursos y capacidades que contribuye a mejorar la eficacia de la } \\
\text { organización" }\end{array}$ \\
Senge, 1993, p. 3) & $\begin{array}{l}\text { Es una organización donde "las personas expanden continuamente su capacidad de } \\
\text { crear los resultados que realmente desean, donde expanden y se nutren los patrones } \\
\text { de pensamiento nuevos, donde la aspiración colectiva queda en libertad, y donde la } \\
\text { gente está continuamente aprendiendo a aprender juntos". }\end{array}$ \\
(Garvin, Edmondson, y Gino, & $\begin{array}{l}\text { Es "un lugar donde los colaboradores tienen éxito en crear, adquirir, y la } \\
\text { transferencia de conocimiento" }\end{array}$ \\
Marquardt (2002, p. 247 $)$ & $\begin{array}{l}\text { "Una empresa que aprende con eficacia y de manera colectiva y continuamente se } \\
\text { transforma para una mejor gestión y uso del conocimiento" }\end{array}$ \\
\hline
\end{tabular}

Fuente: Elaboración Propia

Existen diversas corrientes teóricas que abordan el aprendizaje en las organizaciones, autores como Argyris y Schön (1978) sostienen que el aprendizaje se presenta en las organizaciones por medio de las personas que laboran para ella, y que se constituyen en agentes. Según los autores existe un sistema organizacional de aprendizaje.

Para Senge (1993) el aprendizaje organizacional se compone de cinco disciplinas: el dominio personal, los modelos mentales, una visión compartida, el aprendizaje en equipo y el pensamiento sistémico. 
Por su parte, Marquardt (1996) sostiene que el aprendizaje organizacional se compone principalmente de cinco elementos; el aprendizaje, la organización, las personas, el conocimiento y la tecnología. Además, se encuentran en la literatura autores como Garvin, Edmondson y Gino (2008) quienes enfocan su atención en únicamente tres componentes; el apoyo abierto al aprendizaje, las prácticas y procesos concretos de aprendizaje y el liderazgo que fortalezca el aprendizaje.

La revisión de literatura permite identificar a Watkins y Marsick (1993), como las exponentes principales. Ellas señalan que el aprendizaje organizacional se compone de siete tipos, distintos pero relacionados entre sí, las cuales definen a una organización que aprende, tanto a nivel individual, a nivel de equipo y a nivel de organización. Estos son los siguientes:

\section{a) Creación de oportunidades para un aprendizaje continuo}

Las oportunidades que brinda la organización sirven para establecer las oportunidades de aprendizaje constante para todos sus colaboradores; es por esto, que para cada puesto de trabajo se diseñan las actividades a realizar de tal forma que permitan al colaborador un aprendizaje permanente (Watkins y Marsick, 1993), aquí el aprendizaje está incorporado al trabajo para que las personas puedan aprender en el contexto de trabajo, lo que genera oportunidades de aprendizaje continuo para todos sus miembros.

\section{b) Diálogo y la indagación}

Refleja el esfuerzo de una organización por crear una cultura de debate, de investigación, de realimentación e intercambio, produce que las personas aumenten sus habilidades para expresar sus puntos de vista así como también su capacidad para escuchar y cuestionar el punto de vista de los demás (Watkins y Marsick, 1993).

\section{c) Colaboración y el aprendizaje en equipo}

Este indicador constituye el esfuerzo que se hace para la conformación y preparación de los equipos de trabajo, haciendo énfasis en el aspecto colaborativo, es por esto que las actividades se diseñan de tal forma que los miembros de los equipos puedan aprender de ellos mismos y a trabajar en conjunto (Watkins y Marsick, 1993), aquí el trabajo está diseñado para orientar a los grupos y acceder a los diferentes modos de pensar; se espera que los grupos trabajen y aprendan juntos; la colaboración es valorada por la cultura y recompensada.

\section{d) Captar y compartir el aprendizaje}

Indica los esfuerzos para establecer sistemas para capturar y compartir el aprendizaje. Procesos que poseen las organizaciones para crear y compartir una visión colectiva, se cuenta con sistemas para compartir los aprendizajes (Watkins y Marsick, 1993).

e) Capacitar a las personas hacia una visión colectiva 
Esta dimensión refleja el proceso que sigue una organización para compartir una visión colectiva y conseguir tanto el compromiso como el feedback de sus miembros sobre la diferencia entre lo que se es y la nueva visión, es decir, lo que se desea ser (Watkins y Marsick, 1993) aquí las personas están involucradas en establecer, tener e implementar una visión de conjunto.

\section{f) Conectar a la organización con su entorno}

Las personas comprenden el efecto de su trabajo en toda la empresa, exploran el entorno y utilizan la información para ajustar las prácticas de trabajo, la organización está vinculada a sus comunidades (Watkins y Marsick, 1993).

\section{g) Estimular un liderazgo estratégico para el aprendizaje}

Esta dimensión indica el grado en el cual los líderes utilizan de manera estratégica el aprendizaje para el logro de los objetivos propuestos y se constituyen en modelos a seguir (Watkins y Marsick, 1993).

\section{Compromiso y aprendizaje organizacional}

En la literatura se encuentran estudios que evidencian la relación de estas variables (Che, Kumar y Gua, 2009). Las actitudes de los colaboradores hacia la formación, así como el acceso percibido a la formación, el apoyo social para la formación, la motivación para aprender y los beneficios percibidos de la formación, están positivamente relacionados al compromiso de la organización (Atak, 2011).

Los colaboradores con altos niveles de compromiso con la organización tienden a identificarse más fácilmente con su cultura, sus objetivos y los valores de la misma, además, están dispuestos a aprender cualquier habilidad y captar el conocimiento necesario para alcanzar la meta de la organización (Chang, Tsai y Tsai, 2011).

Cuando existen actividades de aprendizaje que están incorporadas al trabajo para que las personas puedan aprender en el contexto de trabajo y además se le brindan oportunidades de aprendizaje continuo para todos sus miembros, los miembros de la organización afirman su apego a la Organización, generando el deseo de seguir trabajando y además se produce un alineamiento del colaborador con las metas y los valores de la institución (Mehrabi, Jadidi, Allameh y Alemzadeh, 2013)

Por otra parte, cuando en el personal se promueve una cultura de aprendizaje y se les proporciona las oportunidades para llevar a cabo tareas difíciles que permiten el desarrollo de sus competencias, el personal tiene más motivación para realizar un trabajo cooperativo, lo que promueve y desarrolla el compromiso hacia la organización (Usefi, Nazari y Zargar, 2013).

Finalmente, existe evidencia empírica que muestra que ambas variables han sido objeto de estudio en instituciones educativas del nivel superior (Hussain, Ali, Khalid, Shafique y Ahmad, 2016). 


\section{MÉTODOS Y MATERIALES}

El estudio se realizó bajo un enfoque cuantitativo, de tipo correlacional, con diseño no experimental transeccional. Para medir el compromiso organizacional de utilizó la Escala de Compromiso Organizacional (Commitment Scale) de Allen y Meyer (1990). Para medir el aprendizaje organizacional se utilizó el Cuestionario de las Dimensiones del Aprendizaje Organizacional (Dimensions of the Learning Organization Questionnaire) de Watkins y Marsick (1999).

La unidad muestral la constituyó el personal docente de la Universidad Pedagógica Nacional, asignado a los departamentos académicos. El estudio incluyó los diez departamentos académicos de la UPNFM, cinco de la Facultad de Ciencia y Tecnología e igual número de la Facultad de Humanidades. La tabla 3 recoge la distribución de la muestra de docentes de cada facultad y por departamento académico, calculada a partir del total de docentes que actualmente están adscritos a sus departamentos.

Para el análisis de los datos se utilizó SPSS 22.0. Al analizar la confiabilidad del instrumento se observó un Alpha de Cronbach de .912 en la escala de Aprendizaje Organizacional y .832 en la escala del Compromiso Organizacional.

Tabla 3 Población y Muestra

\begin{tabular}{|c|c|c|c|}
\hline Facultad & Deptos. & $\mathrm{N}$ & $\mathrm{n}$ \\
\hline \multirow{10}{*}{ 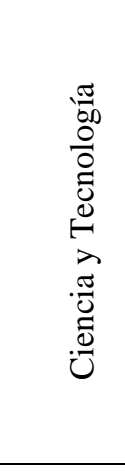 } & Matemáticas & 21 & 16 \\
\hline & Educación & 16 & 12 \\
\hline & Ciencias & & \\
\hline & Naturales & 16 & 9 \\
\hline & Técnicas & 9 & 8 \\
\hline & Industrial & & \\
\hline & Tecnología de & & \\
\hline & Alimentos y & 9 & 8 \\
\hline & Textiles & & \\
\hline & & 71 & 53 \\
\hline & Deptos. & $\mathrm{N}$ & $\mathrm{n}$ \\
\hline \multirow{9}{*}{ 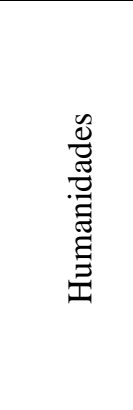 } & $\begin{array}{l}\text { Ciencias de la } \\
\text { Educación }\end{array}$ & 37 & 25 \\
\hline & Ciencias & 21 & 12 \\
\hline & Sociales & 21 & 12 \\
\hline & Letras y & 26 & 14 \\
\hline & Lenguas & 20 & 14 \\
\hline & Educación & 11 & 9 \\
\hline & Física & & \\
\hline & Artes & 8 & 5 \\
\hline & & 103 & 65 \\
\hline
\end{tabular}

Fuente: Elaboración propia 
Para medir la relación de las variables se utilizó el coeficiente de correlación Pearson y para confirmar la relación se observó el valor de significancia $(p)$, para las pruebas de hipótesis se utilizó una $\alpha$ de 0.05.

La hipótesis contrastada en el estudio se describe a continuación.

H1: $r x y=r \neq 0$ (Existe relación entre el Compromiso Organizacional y el Aprendizaje Organizacional).

\section{RESULTADOS}

\section{Datos descriptivos}

En el estudio participaron 53 docentes de la Facultad de Ciencia y Tecnología (44.9\%) y 65 docentes de la facultad de Humanidades (55.1\%). Del total de participantes, 34 son hombres (28.8\%) y 84 son mujeres $(71.2 \%)$.

La tabla 4 recoge los datos descriptivos obtenidos del análisis de información recolectada, para las dos variables que conforman este estudio.

\begin{tabular}{ccc}
\multicolumn{3}{c}{ Tabla 4 Datos descriptivos de las variables } \\
\hline Medida & $\begin{array}{c}\text { Aprendizaje } \\
\text { Organizacional }\end{array}$ & $\begin{array}{c}\text { Compromiso } \\
\text { Organizacional }\end{array}$ \\
\hline $\mathbf{n}$ & 118 & 118 \\
Media & 3.84 & 3.90 \\
Mediana & 3.83 & 4 \\
Moda & 3.83 & 4.28 \\
$\begin{array}{c}\text { Desviación } \\
\text { Estándar }\end{array}$ & 0.468 & 0.487 \\
\hline
\end{tabular}

Fuente: Análisis de datos recolectados

La tabla 5 recoge los datos descriptivos del análisis efectuado sobre la variable compromiso organizacional, de acuerdo a los tres tipos mencionados en este estudio.

Tabla 5 Resultados por tipo de compromiso

\begin{tabular}{lccc}
\hline Medida & Afectivo & Continuidad & Normativo \\
\hline $\mathbf{N}$ & 118 & 118 & 118 \\
Media & 4.16 & 3.74 & 3.82 \\
Mediana & 4.25 & 3.88 & 3.89 \\
Moda & 4.75 & 4.25 & 4.11 \\
Desviación & 0.591 & 0.774 & 0.579 \\
Estándar & & & \\
\hline
\end{tabular}

Fuente: Análisis de datos recolectados

La tabla 6 recoge los datos descriptivos del análisis efectuado sobre la variable aprendizaje organizacional, de acuerdo a los siete tipos que han sido señalados en este estudio. 
Tabla 6 Resultados por tipo de aprendizaje organizacional

\begin{tabular}{|c|c|c|c|c|c|c|c|}
\hline Medida & $\begin{array}{l}\text { Creación de } \\
\text { oportunidades } \\
\text { para el } \\
\text { aprendizaje } \\
\text { continuo }\end{array}$ & $\begin{array}{l}\text { Dialogo e } \\
\text { Indagación }\end{array}$ & $\begin{array}{l}\text { Colaboración y } \\
\text { el aprendizaje } \\
\text { en Equipo }\end{array}$ & $\begin{array}{l}\text { Captar y } \\
\text { compartir el } \\
\text { aprendizaje }\end{array}$ & $\begin{array}{l}\text { Capacitar a } \\
\text { las } \\
\text { personas } \\
\text { hacia una } \\
\text { visión } \\
\text { colectiva }\end{array}$ & $\begin{array}{l}\text { Conectar a la } \\
\text { organización } \\
\text { con su } \\
\text { entorno }\end{array}$ & $\begin{array}{l}\text { Estimular el } \\
\text { Liderazgo } \\
\text { Estratégico } \\
\text { para el } \\
\text { aprendizaje }\end{array}$ \\
\hline $\mathbf{n}$ & 118 & 118 & 118 & 118 & 118 & 118 & 118 \\
\hline Media & 4.09 & 4.20 & 3.90 & 3.72 & 3.47 & 3.69 & 3.82 \\
\hline Mediana & 4.17 & 4.33 & 4.00 & 3.83 & 3.50 & 3.83 & 3.92 \\
\hline Moda & 4.17 & 4.00 & 4.00 & 4.33 & 3.33 & 3.67 & 4.17 \\
\hline $\begin{array}{l}\text { Desviación } \\
\text { Estándar }\end{array}$ & 0.503 & 0.512 & 0.638 & 0.731 & 0.743 & 0.682 & 0.634 \\
\hline
\end{tabular}

Fuente: Análisis de datos recolectados

La tabla 7 ofrece los datos descriptivos obtenidos para las variables del estudio con la muestra de la Facultad de Ciencia y Tecnología.

Tabla 7 Datos descriptivos de las variables para la Facultad de Ciencia y Tecnología

\begin{tabular}{lcc}
\hline Medida & $\begin{array}{c}\text { Aprendizaje } \\
\text { Organizacional }\end{array}$ & $\begin{array}{c}\text { Compromiso } \\
\text { Organizacional }\end{array}$ \\
\hline $\mathbf{N}$ & 53 & 53 \\
Media & 3.82 & 3.93 \\
Mediana & 3.90 & 4.12 \\
Moda & 4.10 & 4.28 \\
Desviación & 0.504 & 0.507 \\
Estándar & & \\
\hline
\end{tabular}

Fuente: Análisis de datos recolectados

La tabla 8 ofrece los datos descriptivos obtenidos para la Facultad de Humanidades.

Tabla 8 Datos descriptivos de las variables para la Facultad de Humanidades

\begin{tabular}{ccc}
\hline Medida & $\begin{array}{c}\text { Aprendizaje } \\
\text { Organizacional }\end{array}$ & $\begin{array}{c}\text { Compromiso } \\
\text { Organizacional }\end{array}$ \\
\hline $\mathbf{N}$ & 65 & 65 \\
Media & 3.86 & 3.88 \\
Mediana & 3.83 & 3.92 \\
Moda & 3.83 & 3.88 \\
$\begin{array}{c}\text { Desviación } \\
\text { Estándar }\end{array}$ & 0.439 & 0.472 \\
\hline
\end{tabular}

Fuente: Análisis de datos recolectados

\section{Efecto del Compromiso en el Aprendizaje Organizacional}

A continuación se describen los coeficientes de determinación de Pearson para medir el efecto de la variable independiente sobre la dependiente. Los datos de la tabla 9 y 10 muestran la influencia del 
Compromiso sobre el Aprendizaje Organizacional, se observa un $r: 0.718$ y un $\mathrm{R}^{2}$ de 0.516 a un valor $p$ : $0.000<\alpha: 0.05$. Esto indica que el cambio en los niveles de compromiso que muestran los docentes de la UPNFM afecta su capacidad de aprendizaje.

La tabla 11 y 12 muestran la influencia que tiene el Compromiso sobre el Aprendizaje Organizacional en la Facultad de Ciencia y Tecnología, se observa un $r: 0.762$ y un $\mathrm{R}^{2}$ de 0.581 a un valor $p: 0.000<\alpha$ : 0.05. De aquí se infiere que el cambio en los niveles de compromiso que muestran los docentes de la Facultad de Ciencia y Tecnología afecta su capacidad de aprendizaje.

Asimismo, la tabla 13 y 14 muestran la influencia que tiene el Compromiso sobre el Aprendizaje Organizacional en la Facultad de Humanidades, se observa un $r: 0.684$ y un $\mathrm{R}^{2}$ de 0.468 a un valor $p$ : $0.000<\alpha$ : 0.05 . Estos datos señalan que el cambio en los niveles de compromiso que muestran los docentes de la Facultad de Humanidades afecta su capacidad de aprendizaje, esta relación es menor en comparación con la de la Facultad de Ciencia y Tecnología.

Tabla 9 Influencia de Compromiso en el Aprendizaje Organizacional

\begin{tabular}{ccccc}
\hline Modelo & $\mathrm{R}$ & R cuadrado & $\begin{array}{c}\text { R cuadrado } \\
\text { ajustado }\end{array}$ & $\begin{array}{c}\text { Error estándar de } \\
\text { la estimación }\end{array}$ \\
\hline 1 & .718 & .516 & .512 & .32683 \\
\hline \multicolumn{5}{c}{ Fuente: Análisis de datos Recolectados }
\end{tabular}

Tabla 10 ANOVA de la Influencia de Compromiso en el Aprendizaje Organizacional

\begin{tabular}{llrrrrr}
\hline Modelo & & $\begin{array}{c}\text { Suma de } \\
\text { cuadrados }\end{array}$ & Gl & \multicolumn{2}{c}{$\begin{array}{c}\text { Media } \\
\text { cuadrática }\end{array}$} & F \\
\hline 1 & Regresión & 13.200 & 1 & 13.200 & 123.576 & .000 \\
& Residuo & 12.391 & 116 & .107 & & \\
& Total & 25.591 & 117 & & & \\
\hline
\end{tabular}

Fuente: Análisis de datos Recolectados

Tabla 11 Influencia de Compromiso en el Aprendizaje Organizacional en la Facultad de Ciencia y Tecnología

\begin{tabular}{ccccc}
\hline Modelo & $\mathrm{R}$ & R cuadrado & $\begin{array}{c}\text { R cuadrado } \\
\text { ajustado }\end{array}$ & $\begin{array}{c}\text { Error estándar de } \\
\text { la estimación }\end{array}$ \\
\hline 1 & .762 & .581 & .573 & .32931 \\
\hline \multicolumn{5}{c}{ Fuente: Análisis de datos Recolectados }
\end{tabular}

Tabla 12 ANOVA de la Influencia de Compromiso en el Aprendizaje Organizacional en la Facultad de Ciencia y

\begin{tabular}{llrrrrr}
\multicolumn{7}{c}{ Tecnología } \\
\multicolumn{1}{c}{} & \multicolumn{1}{c}{$\begin{array}{c}\text { Suma de } \\
\text { cuadrados }\end{array}$} & Gl & $\begin{array}{c}\text { Media } \\
\text { cuadrática }\end{array}$ & F & Sig. \\
\hline 1 & Regresión & 7.682 & 1 & 7.682 & 70.835 & .000 \\
& Residuo & 5.531 & 51 & .108 & & \\
& Total & 13.212 & 52 & & & \\
\hline
\end{tabular}

Fuente: Análisis de datos Recolectados 
Tabla 13 Influencia de Compromiso en el Aprendizaje Organizacional en la Facultad de Humanidades

\begin{tabular}{ccccc}
\hline Modelo & $\mathrm{R}$ & R cuadrado & $\begin{array}{c}\text { R cuadrado } \\
\text { ajustado }\end{array}$ & $\begin{array}{c}\text { Error estándar de } \\
\text { la estimación }\end{array}$ \\
\hline 1 & .684 & .468 & .460 & .32237 \\
\hline \multicolumn{5}{c}{ Fuente: Análisis de datos Recolectados } \\
\hline
\end{tabular}

Fuente: Análisis de datos Recolectados

Tabla 14 ANOVA de la Influencia de Compromiso en el Aprendizaje Organizacional

\begin{tabular}{llrrrrr}
\hline Modelo & & $\begin{array}{c}\text { Suma de } \\
\text { cuadrados }\end{array}$ & Gl & \multicolumn{1}{c}{ Media } \\
cuadrática & F & Sig. \\
\hline 1 & Regresión & 5.767 & 1 & 5.767 & 55.490 & .000 \\
& Residuo & 6.547 & 63 & .104 & & \\
& Total & 12.314 & 64 & & & \\
\hline
\end{tabular}

Fuente: Análisis de datos Recolectados

\section{DISCUSIÓN Y CONCLUSIONES}

\section{Análisis descriptivo}

Los hallazgos del estudio mostraron una mediana y moda de 4 y 4.28 respectivamente (ver tabla 4 ) en el Compromiso Organizacional, lo que explica que el 50\% de los datos observados se ubican por encima y la diferencia por debajo de ese valor. Estos resultados difieren a los hallazgos de Ochoa (2014) quien estudio esta variable en el contexto de una universidad pública de Honduras, el autor encontró una mediana de 3.77, al comparar ambos resultados se observa que los valores mostrados en la UPNFM son más favorables respecto a los encontrados por el autor, los hallazgos de este estudio sugieren que las respuesta más común según la escala propuesta es el "De acuerdo", es decir, aunque no como se espera, los docentes continúan laborando para la Universidad y están dispuestos a dedicar un mayor esfuerzo para lograr los objetivos lo que ayuda a mejorar el rendimiento y la eficiencia tanto a nivel individual, de grupo, así como a nivel de la Organización (Allen y Meyer, 1997).

Para Adekola (2012) los docentes de las universidades públicas deben mostrar un alto compromiso con su institución, lo cual es coherente si se considera el hecho que la estabilidad laboral en las universidades públicas en superior a las del sector privado. Los docentes de las universidades públicas se sienten seguros sobre su continuidad en el trabajo lo que evita que estén continuamente buscando otra oportunidad laboral, por tanto el no tener la presión de generar ganancia y la demanda constante de cumplir con los objetivos, permite una mayor libertad a los docentes de las universidades públicas, sin el temor que vayan a ser despedidos por no lograr las metas de la organización (Meyer, et al., 2002).

Durante el análisis de los tres tipos que comprenden el Compromiso Organizacional, en el compromiso afectivo se observó una mediana y moda de 4.25 y 4.75 respectivamente, ambos valores cercanos al "de acuerdo" según la escala, estos resultados son coherente con los de Ochoa (2014), en ambas universidades 
se observa que existe un deseo de los docentes en continuar trabajando muestra un fuerte apego emocional, abierto a participar y a identificarse con los objetivos y valores de la Universidad (Allen y Meyer, 1997; Meyer y Allen, 1991).

En el compromiso continuidad, se observó una mediana y moda de 3.88 y 4.25 respectivamente, ambos valores cercanos al "de acuerdo" según la escala, con estos resultados existe una aceptación de los docentes que los costos son elevados al dejar de laborar para Universidad y que existen pocas alternativas u oportunidades de trabajo (Allen y Mayer, 1997; Meyer y Allen, 1991).

Ahora bien, en el compromiso normativo se observó una mediana y una moda de 3.89 y 4.11 respectivamente, ambos valores cercanos al "de acuerdo" según la escala, resultados se traducen en una fuerte obligación de los docentes de permanecer en la Universidad, no se atreverían a dejar su trabajo porque sienten que es algo "moralmente incorrecto dejar de laborar en la institución" (Meyer y Parfyonova, 2010), así también muestran valores arraigados en su conducta y sentirían miedo de lo que dirán sus compañeros si abandonan su trabajo (Meyer y Allen, 1991).

Según la teoría propuesta por Meyer y Allen, 1991), los docentes de la UPNF, muestran un compromiso de permanecer más tiempo, una mayor participación en los quehaceres de la Universidad, están propensos a faltar menos días de trabajo, además es probable que se dediquen más en su trabajo, lo que repercute positivamente en la calidad del trabajo que realiza en el aula de clases.

Respecto al aprendizaje organizacional, el estudio mostró una mediana de 3.83 y una moda de igual valor (ver tabla 4), estos resultados son similares a los obtenidos por Ochoa (2014) en donde observó una mediana de 3.86, los resultados muestran que ambas Universidades no muestra valores deseados de aprendizaje

Al analizar cada uno de los tipos de aprendizaje se encontraron valores bajos, por ejemplo, "Capacitar a las personas hacia una visión colectiva" muestra una mediana y moda de 3.5 y 3.33 respectivamente, cabe mencionar que Ochoa (2014) encontró valores similares en la misma dimensión con una mediana y moda de 3.67 y 3.50 respectivamente, estos resultados reflejan que la Universidad muestra debilidades en el proceso que sigue para poder lograr una visión colectiva (Watkins y Marsick,1999), al parecer no se reconoce cuando los docentes toman la iniciativa, ofrece limitadas opciones para ellos decidan las forma de hacer su trabajo, no los hace partícipe cuando se formula la visión de la Universidad, los docentes tienen limitado control sobre los recursos que necesitan para realizar llevar a cabo sus labores plenamente, ellos consideran que no se les apoya cuando asumen riesgos calculados y que les parece que la visión de la Universidad no se elabora tomando en cuenta los diferentes niveles y grupos de trabajo. 


\section{Prueba de hipótesis}

A partir de los datos de la tabla 9 y 10 se infiere, por un lado, que los cambios que se presentan en el aprendizaje organizacional son explicados en un $51.6 \%$ por los cambios que se generan en el compromiso Organizacional, en segundo lugar, dado que p: $0.000<\alpha$ : 0.05 existe evidencia estadísticamente significativa para aceptar la hipótesis y concluir que sí existe influencia del Compromiso Organizacional sobre el Aprendizaje Organizacional.

Los datos de la 11 y 12 muestra la influencia del Compromiso Organizacional sobre el Aprendizaje en la Facultad de Ciencia y Tecnología, que los cambios que se presentan en el aprendizaje organizacional son explicados en un $58.1 \%$ por los cambios que se generan en el compromiso Organizacional, en segundo lugar, dado que p: $0.000<\alpha$ : 0.05 existe evidencia estadísticamente significativa para aceptar la hipótesis y concluir que si existe influencia.

Asimismo, la tabla 13 y 14 presenta los efectos del Compromiso sobre el Aprendizaje en la Facultad de Humanidades, se observó que el Aprendizaje es explicado en un $46.8 \%$ por los cambios que se generan en el Compromiso, dado que p: $0.000<\alpha$ : 0.05 existe evidencia estadísticamente significativa para aceptar la hipótesis y que en efecto, el compromiso organizacional afecta al aprendizaje organizacional.

Los resultados tanto a nivel general como nivel particular en cada facultad, apoyan la teoría propuesta por Bhatnagar (2007) quien considera que el mantener empleados comprometidos mejora la capacidad de la organización para aprender, otros autores también apoyan esta teoría (Harel y Tzafrir,1999). Para Cho y Kwon (2005), la organización que tiene empleados comprometidos están motivados y presentan mayores deseos de aprender en su lugar de trabajo.

El Compromiso de los docentes se relaciona positivamente con el Aprendizaje cuando a los docentes se les deja expresar sus puntos de vista y adquieren la capacidad de escuchar e indagar las opiniones de los otros y el trabajo se diseña para acceder a los diferentes modos de pensar, (Nazari, 2013; Mehrabi, 2013; Kamis y Noermijati, 2013).

Finalmente, el estudio demuestra que en la medida que se aumenta el sentimiento de apego del docente hacia la Universidad, sus objetivos personales e institucionales se alinean y se le incluye en la tomas de decisiones importantes, en medida se fomentan espacios que generan acuerdos para el aprendizaje individual y colectivo, lo que conduce a mejorar la forma de aprender a nivel de Universidad.

\section{RECOMENDACIONES}

A continuación se describen las acciones que podría emprender la UPNFM para mantener y mejorar el Compromiso y el Aprendizaje Organizacional. Respecto al Compromiso Organizacional se sugiere que la 
Universidad muestre interés a los problemas que se le presentan a sus docentes en el desarrollo de sus actividades.

En cuanto al Aprendizaje Organizacional, la Universidad debe premiar el aprendizaje y debe conceder cierta autonomía, con parámetros definidos, en la toma de decisiones, y en el acceso a los recursos necesarios para fortalecer el aprendizaje, debe fomentar la planificación colaborativa y flexible, en donde se involucre al personal docente, debe valorar y recompensar la colaboración en equipo, debe contar con sistemas que les permita identificar, registrar, actualizar y usar de forma eficiente las capacidades de sus docentes, debe alentar a sus docentes a compartir sus experiencias y capacidades con sus colegas, comprender la importancia que tienen los líderes (Jefes de Departamento, Decanos, Vicerrectoría, Rectoría) para modelar y apoyar el aprendizaje, debe generar procesos de entrenamiento producto de las necesidades y limitaciones que presenta el personal, debe promover la participación de sus docentes en los procesos de toma de decisiones, debe promover una cultura de adquisición, creación, transferencia e intercambio de conocimientos como valores fundamentales de la Universidad, debe generar espacios de equilibrio laboral y familiar en sus docentes y debe mostrar interés por conocer la opinión de la comunidad, tomándola en cuenta al planificar y al tomar decisiones.

\section{REFERENCIAS BIBLIÓGRAFICAS}

Allen, N. J., y Meyer, J. P. (1990). The measurement and antecedents of affective, continuance, and normative commitment. Journal of Occupational Psychology, 63(1), 1-18.

Argyris, C., y Schön, D. (1978). Organizational learning: A theory of action perspective, Reading, MA: Addison-Wesley Publishing.

Atak, M. (2011). A Research on the Relation between Organizational Commitment and Learning Organization. African Journal of Business Management. 5(14), 5612-5616.

Bhatnagar, J. (2007). Predictors of organizational commitment in India: strategic HR roles, organizational learning capability and psychological empowerment. The International Journal of Human resource Management, 18(10), 1782-1811.

Chang, C., C., Tsai, M., C., Tsai, M., S. (2011). Influences of the Organizational Citizenship Behaviors and Organizational Commitments on the Effects of Organizational Learning in Taiwan. International Conference on E-business, Management and Economics, 3, 1-5.

Che Rose, R. Kumar. Gua Pak.O. (2009). The Effect of Organizational Learning on Organizational Commitment, Job Satisfaction and Work Performance. The Journal of Applied Business Research, 25(6).

Cho, D. Y., y Kwon, D. B. (2005). Self-directed learning readiness as an antecedent of organizational commitment: a Korean study. International Journal of Training y Development, 9(2), 140-152. 
Fernández de Morgado, N. (2008). Aprendizaje Organizacional en la Universidad Simón Bolívar según gerencia media: Comparación entre dos divisiones. Paradigma, 24 (2), 55-80.

Garvin, D. A., Edmondson, A. C., y Gino, F. (2008). Is yours a learning organization? Harvard Business Review, 86(3), 109-116.

Greenberg, Jerald, y Baron, Robert A. (2003). Behavior in organizations, 8th ed., Prentice-Hall.

Harel, G. H., y Tzafrir, S. S. (1999). The effect of human resource management practices on the perceptions of organizational and market performance of the firm. Human Resource Management, $38,185-200$.

Hussain, Muhammad Wajahat; Ali, Ahsan; Khalid, Mehran; Shafique, Muhammad Nouman y Ahmad, Hussain (2016). Organizational Silence A Predictor of Organizational Commitment in Higher Education Institution. Developing Country Studie. 6 (2), 123-128.

Joo, B. K. y Park, S. (2010), "Career satisfaction, organizational commitment, and turnover intention: The effects of goal orientation, organizational learning culture and developmental feedback", Leadership y Organization Development Journal, 31(6), 482-500.

Kamis, R., A.,Noermijati, C., S. (2013). The Influence of Organizational Commitment and Individual Competence on Teacher Performance: In the Learning Organization Perspective. (A Study on Elementary School Teachers in Ternate City). International Journal of Business and Behavioral Sciences, 3(8), 19-35.

Lee, K., Allen. N.J., Meyer, J.P., y Rhee, K-Y. (2001). "The Three component model of organizational commitment: An application to South Korea". Applied Psychology: An International Review, 50(4): $596-614$.

Marquardt, M (1996). Building the learning organization. New York: McGraw-Hill

Marquardt, M. (2002). Building the learning organization: Mastering the 5 elements for corporate learning. Palo Alto, CA: Davies-Black Publishing.

Mehrabi, J., Jadidi, M., Allameh, F. y Alemzadeh, M., (2013). The Relationship between Organizational Commitment and Organizational Learning (Boroojerd Telecommunication Company as Case Study). International Journal of Academic Research in Business and Social Sciences, 1 (3), 130-139.

Meyer, J. P. y Allen, N. J. (1987). A longitudinal analysis of the early development and consequences of organizational commitment. Canada Journal Behavioral Science, 19, 199-215.

Meyer, J. P., Allen, N. J., y Smith, C. A. (1993). Commitment to organizations and occupations: Extension and test of a three-component conceptualization. Journal of Applied Psychology, 78, 538551. 
Meyer, J. P., and Allen, N. J., (1991). Three Component Conceptualization of Organizational Commitment. Human Resource Management Review, 1(1), 61-89.

Meyer, J. P., and Parfyonova, N. M., (2010). Normative commitment in the workplace: A theoretical analysis and re-conceptualization. Human Resource Management Review, 20(10), 283-294.

Meyer, J.P. y Allen, N.J. (1984). 'Testing the side bet theory of organizational commitment: some methodological considerations', Journal of Applied Psychology, 69, 372-378.

Meyer, J.P., Allen, N.J. y Gellatly, I.R. (1990). Affective and continuance commitment to the organization: evaluation of measures and analysis of concurrent and time lagged relations, Journal of Applied Psychology, 75, 710-720.

Mowday, R.T., Steers, R.M., y Porter, L.W. (1979). "Measurement of organizational commitment". Journal of Vocational Behavior, 14(3): 224-247.

Newstrom, J. W. and Davis, K. (2002). Organizational Behavior. "Human Behavior at Work." 11th Edition: New Delhi Tata McGraw - Hill Publishing Company Limited.

Ochoa Cervantez, D. (2014). Compromiso, Aprendizaje y Cultura Organizacional y su Relación con Calidad del Servicio Percibido en Instituciones de Educación Superior de Honduras: Un Estudio de Casos. (Tesis inédita de Doctorado). Universidad Católica de Honduras, San Pedro Sula, Honduras.

Rashid, M.Z.A., Sambasivan, M., y Johari, J. (2003). The influence of corporate culture and organizational commitment and performance. Journal of Management Development, 22, 708- 728.

Senge, P. M., (1993). The Fifth Discipline: The Art and Practice of the Learning Organization: Book review.

Usefi, S., Nazari, R., y Zargar, T. (2013). The Relationship between Organizational Learning and Organizational Commitment in Sport Organizations. Management and Administrative Sciences Review, 2(6), 682-688.

Watkins, K. and Marsick, V. (1999), Dimensions of the Learning Organization Questionnaire, Partners for the Learning Organization, Warwick, RI.

Watkins, K. E., and Marsick, V. J. (1993). Sculpting the learning organization Lessons in the art and science of systematic change: San Francisco: Jossey- Bass. 